\title{
Media Flow Rate Allocation in Multipath Networks
}

\author{
Dan Jurca, Student Member, IEEE, and Pascal Frossard, Senior Member, IEEE
}

\begin{abstract}
We address the problem of joint path selection and source rate allocation in order to optimize the media specific quality of service in streaming of stored video sequences on multipath networks. An optimization problem is proposed in order to minimize the end-to-end distortion, which depends on video sequence dependent parameters, and network properties. An in-depth analysis of the media distortion characteristics allows us to define a low complexity algorithm for an optimal flow rate allocation in multipath network scenarios. In particular, we show that a greedy allocation of rate along paths with increasing error probability leads to an optimal solution. We argue that a network path shall not be chosen for transmission, unless all other available paths with lower error probability have been chosen. Moreover, the chosen paths should be used at their maximum available end-to-end bandwidth. Simulation results show that the optimal flow rate allocation carefully adapts the total streaming rate and the number of chosen paths, to the end-to-end transmission error probability. In many scenarios, the optimal rate allocation provides more than $20 \%$ improvement in received video quality, compared to heuristic-based algorithms. This motivates its use in multipath networks, where it optimizes media specific quality of service, and simultaneously saves network resources at the price of a very low computational complexity.
\end{abstract}

Index Terms-Multipath networks, path selection, rate allocation, video distortion.

\section{INTRODUCTION}

W ITH the development of novel network infrastructures and increasing available bandwidth, multimedia applications over the Internet become attractive for both businesses and home users. Fast deployment of broadband last-mile connections, increase in wireless coverage of remote living areas, and the long awaited debut of $3 \mathrm{G}$ wireless services offer as many inter-operable communication solutions.

However, the viability of a streaming application mostly depends on its ability to meet stringent requirements (e.g., controlled error rate and low delay) and on medium and long term stability of the transport infrastructure. As the Internet is still far from providing any widely deployed guarantee of service solution, efficient media streaming strategies have to be devised to get the best out of the network infrastructure. Lately, multipath streaming emerged as a valid solution to overcome some of the lossy Internet path limitations [1], [2]. It allows for an increase in streaming bandwidth, by balancing the load over mul-

Manuscript received March 13, 2006; revised March 18, 2007. This work was supported by the Swiss NSF under Grant PP002-68737. The associate editor coordinating the review of this manuscript and approving it for publication was Dr. Deepak S. Turaga.

The authors are with the Ecole Polytechnique Fédérale de Lausanne (EPFL), Signal Processing Institute, CH-1015 Lausanne, Switzerland (e-mail: dan.jurca@epfl.ch; pascal.frossard@epfl.ch).

Color versions of one or more of the figures in this paper are available online at http://ieeexplore.ieee.org.

Digital Object Identifier 10.1109/TMM.2007.902852 tiple network paths between the media server and the client. It also provides means to limit packet loss effects, when combined with error resilient streaming strategies and scalable encoding capabilities of the latest encoding standards [3]-[6]. Most of the research work dedicated to multipath streaming focuses on the process itself (media caching and scheduling aspects), but generally not towards finding which paths should ideally be used for the streaming application, for a given network topology between a streaming server and a client. These works rely on classic routing algorithms that find the best path (or set of paths) given some established network metrics. While this may be optimal in terms of network utilization, it is certainly suboptimal from the viewpoint of the media streaming application. In $30-80 \%$ of the cases, the best paths found by classic routing algorithms are suboptimal from a media perspective [7].

This work proposes to address the problem of streaming path allocation in a multipath network, which takes into account media aware metrics during the decision process. The early work in [8] derives a few empirical rules on what paths should be considered by the streaming application, based on experimental data. These rules consider network metrics (e.g., available bandwidth, loss rate, and hop distance), and other media aware metrics (e.g., link jointness/disjointness, video distortion). Our work provides a more general framework for the analysis of joint path selection and flow rate allocation in multipath streaming, driven by media-specific metrics. We consider a multipath network model that supports the partitioning of a media sequence into multiple media flows. We further assume that the streaming server that can perform simple adaptation of the streaming rate of pre-encoded packet media streams (by packet filtering, or by taking advantage of scalable coding, for example). A generic video distortion metric is proposed, which encompasses both the source distortion that is mostly driven by the streaming rate, and the channel distortion that depends on the loss probability.

Finding the optimal flow rate allocation in multipath networks is a very complex problem in generic scenarios. However, in our specific scenario, we show that a careful analysis of the video distortion evolution allows to derive a linear complexity algorithm for the joint optimal path selection, and flow rate allocation. In other words, our main objective is to jointly find i) the optimal streaming rate for a given, pre-encoded video packet stream so that the quality at receiver is maximized and, ii) which network paths should be used for relaying the video stream to the client. Interestingly enough, our conclusions demonstrate that the answer to these two questions is represented by a careful tradeoff among available network bandwidth (translated into video streaming rate), transmission loss process, and number of utilized paths. And, in contrary to the commonly admitted opinion, flooding the network in pushing the streaming rate to the limits the total available bandwidth, rarely provides efficient 


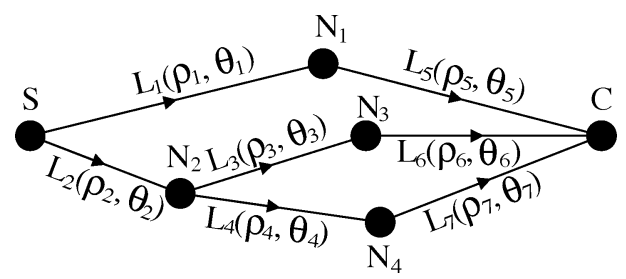

Fig. 1. Multipath network scenario.

strategies in the absence of complex and expensive transcoding strategies for stored video streams.

The main contributions of this paper can be briefly summarized as follows.

- We propose a general framework for streaming of pre-encoded media data in multipath networks, which encompasses network and media aware metrics.

- We perform the first theoretical media flow analysis on the optimality of number, and choice of network paths, in terms of end-to-end Quality of Service.

- We provide a linear time media aware routing algorithm that outputs the optimal set of network paths to be used in the streaming process, along with the corresponding flow rate distribution.

The paper is organized as follows: Section II presents the streaming framework and formulates our optimization problem. The theoretical analysis of the streaming process is developed in Section III. Section IV presents the routing algorithm and Section V presents our main results. We present the related work in Section VI, and conclude the paper in Section VII.

\section{Distortion Optimized Multipath Media Streaming}

\section{A. Multipath Network Model}

We consider a framework where the media streaming application uses a multipath network, which can be represented as follows. The available network between a media server $S$ and a client $C$ is modeled as a graph $G(V, E)$, where $V=\left\{N_{i}\right\}$ is the set of nodes in the network, and $E$ is the set of links or segments (see Fig. 1). Each link $L_{u}=\left(N_{i}, N_{j}\right) \in E$ connecting nodes $N_{i}$ and $N_{j}$ has two associated positive metrics

- available bandwidth $\rho_{u}>0$ expressed in some appropriate unit (e.g., kbps);

- average loss probability $\theta_{u} \in[0,1]$, assumed to be independent of the streaming rate.

Let $\mathcal{P}=\left\{P_{1}, \ldots, P_{n}\right\}$ denote the set of available loop-free paths between the server $S$ and the client $C$ in $G$, with $n$ the total number of nonidentical end-to-end paths. A path $P_{i}=$ $\left(S, N_{i}, N_{j}, \ldots, C\right)$ is defined as an ordered list of nodes and their connecting links, such that no node appears more than once, and that each link $L_{u}$ between two consecutive nodes in the path belongs to the set of segments $E$. Let further $b_{i}$ and $p_{i}$ denote respectively the end-to-end bandwidth and loss probability of path $P_{i}$. We define the bandwidth of an individual path $P_{i}$ as the minimum of the bandwidths among all links on the path (i.e., the "bottleneck bandwidth"). Hence, we have

$$
b_{i}=\min _{L_{u} \in P_{i}}\left(\rho_{u}\right) .
$$

Under the commonly accepted assumption that the loss process is independent on two consecutive segments, the end-to-end loss probability on path $P_{i}$ becomes a multiplicative function of the individual loss probabilities of all segments composing the path. It can be written as

$$
p_{i}=1-\prod_{L_{u} \in P_{i}}\left(1-\theta_{u}\right) .
$$

Finally, the media application sends data at rate $r_{i}$ on path $P_{i}$, with a cost $c_{i}$. The cost represents the price to be paid by the streaming application, for using path $P_{i}$. As, in general, the underlying transport medium should be transparent for the application, we define the cost function as dependent only on the total flow rate $r_{i}$ sent by the application on path $P_{i}$. A linear cost relation is simply expressed as follows:

$$
c_{i}= \begin{cases}k \cdot r_{i}, & \text { if } P_{i} \text { is used, with } r_{i} \leq b_{i} \\ 0, & \text { if } P_{i} \text { is not used }\end{cases}
$$

where $k$ is a constant (i.e., the cost factor is identical for any path $P_{i}$ in $\mathcal{P}$ ). In this network model, efficient streaming strategies have to carefully allocate the rate between the different network paths. The goal of the next sections is to get the best out of the multipath network, both in terms of cost, and from a mediadriven quality of service perspective.

\section{B. From Network Graph to Flow Tree}

In order to study the flow rate allocation problem in multipath networks, we use a flow tree representation of the network graph $G$. The media server becomes the root of the tree, and each flow $\mathcal{F}_{i}$ represents the share of the overall media stream, which is sent on a network path $P_{i}$. The media stream is the composition of individual media flows, and the client is represented as a set of leaf nodes, with one leaf per flow. Note that several methods in graph theory have been proposed for constructing such trees, and we rather concentrate in this paper on the rate allocation problem, among the branches of the tree. In this case, the rate allocation becomes a flow assignment problem.

Considering that there is (at most) one flow for each network path $P_{i}$, we can transform the original network graph $G$ into a flow tree by duplicating any network edge and vertex that is shared by more than one network path, as represented in Fig. 2. Since the transformation from paths to flows is bijective, each flow is characterized by a maximal end-to-end streaming rate, and an end-to-end loss probability, as computed in Section II-A. The flow $\mathcal{F}_{i}$ on path $P_{i}$ uses a streaming rate $r_{i} \leq b_{i}$, with a loss probability $p_{i}$, and a cost $c_{i}=k \cdot r_{i}$.

Due to the assumption of rate independent loss process, any two flows in the tree are independent in terms of loss probability. However, flows may be dependent in terms of aggregated bandwidth, since they may share joint bottleneck links. The flow tree representation allows us to explicit the constraints imposed on a valid rate allocation. These constraints are imposed by bandwidth limitation on the network links, and flow conservation in the network nodes. The necessary and sufficient conditions for the flow tree model to be a valid representation of the original network graph can finally be grouped into single flow, and multiple flow constraints, and expressed as follows. 


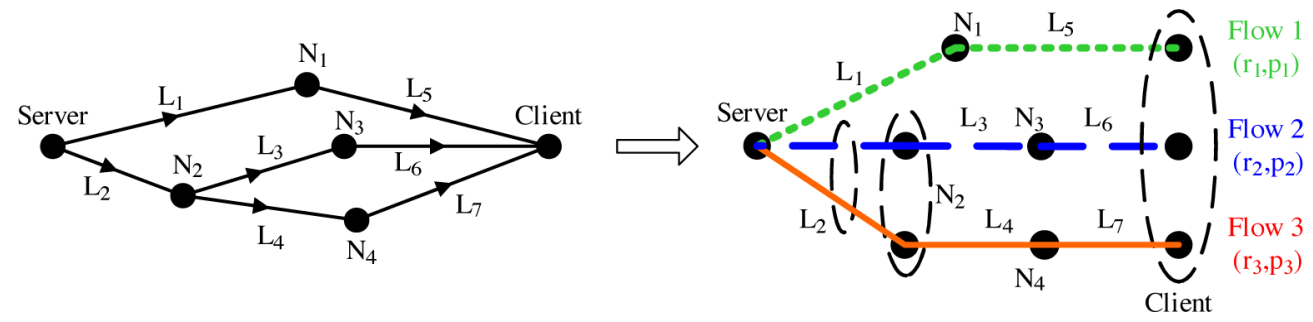

Fig. 2. Equivalent transformation between a network graph and a tree of paths between the server and the client.

1) Single Flow Constraints:

- path bandwidth limitations: $r_{i} \leq b_{i}, \forall P_{i} \in \mathcal{P}$;

- flow conservation at intermediate nodes: for every node $N_{j} \in P_{i}, r_{i}^{i n}=r_{i}^{\text {out }}=r_{i}$, where $r_{i}^{i n}$ and $r_{i}^{o u t}$ are the incoming and respectively outgoing rates of $\mathcal{F}_{i}$ passing through node $N_{j}$.

2) Multiple Flow Constraints:

- link bandwidth limitations:

$$
\sum_{P_{i}: L_{u} \in P_{i}} r_{i} \leq \rho_{u}, \forall L_{u} \in E
$$

- flow conservation at intermediate nodes: for every node $N_{j} \in V$ :

$$
\sum_{P_{i}} r_{i}^{\text {in }}=\sum_{P_{i}} r_{i}^{\text {out }}=\sum_{P_{i}} r_{i}, \forall P_{i}: N_{j} \in P_{i}
$$

\section{Media-Driven Quality of Service}

The end-to-end distortion, as perceived by the media client, can generally be computed as the sum of the source distortion, and the channel distortion. In other words, the quality depends on both the distortion due to a lossy encoding of the media information, and the distortion due to losses experienced in the network. The source distortion $D_{S}$ is mostly driven by the source or streaming rate $R$, and the media sequence content, whose characteristics influence the performance of the encoder (e.g., for the same bit rate, the more complex the sequence, the lower the quality). The source distortion decays with increasing encoding rate; the decay is quite steep for low bit rate values, but it becomes very slow at high bit rate. The channel distortion $D_{L}$ is dependent on the average loss probability $\pi$, and the sequence characteristics. It is roughly proportional to the number of video entities (e.g., frames) that cannot be decoded correctly, and an increase in loss probability augments the channel distortion $D_{L}$. Overall, the end-to-end distortion can thus be written as

$$
D=D_{S}+D_{L}=f(R, \pi, \Gamma)
$$

where $\Gamma$ represents the set of parameters that describe the media sequence. This generic distortion model is quite commonly accepted, as it can accommodate a variety of streaming scenarios. For example, when error correction is available, the total streaming rate has to be split between the video source rate that drives the source distortion $D_{S}$ and the channel rate, which directly influence the video loss rate $\pi$ [9].

The total streaming rate $R$, and the end-to-end loss probability $\pi$ directly depend on the path selection, and the flow rate allocation. In the multipath scenario described before, the media application uses rate allocation $\vec{R}=\left[r_{1}, \ldots r_{n}\right]$, where the flow rate $r_{i}$, with $0 \leq r_{i} \leq b_{i}$, represents the streaming rate on path $P_{i} \in \mathcal{P}$. The total media streaming rate $R$ is expressed as

$$
R=\sum_{i=1}^{n} r_{i} \leq \sum_{i=1}^{n} b_{i}
$$

The overall loss probability $\pi$ experienced by the media application can be computed as the average of the loss probabilities of the $n$ paths

$$
\pi=\frac{\sum_{i=1}^{n} p_{i} \cdot r_{i}}{\sum_{i=1}^{n} r_{i}} .
$$

It is important to note that increasing $R$ with the addition of a path reduces the source distortion. However, the addition of a path generally impacts the loss probability $\pi$, and may augment the channel distortion. The optimal flow rate allocation therefore results from a trade-off between increase the streaming rate, and controlling the end-to-end loss probability. Finally, since paths may not be completely disjoint, $\vec{R}$ is a valid rate allocation on the network graph $G$, if and only if $G$ can simultaneously accommodate the flow rates on all paths in $\mathcal{P}$. A necessary condition for the equality in the right side of (5) to be verified requires that all bottleneck links of the $n$ streaming paths are disjoint. Sufficient conditions for valid rate allocation are analyzed in the next section.

\section{Multipath Rate Allocation: Problem Formulation}

We consider the problem of the optimal routing and rate allocation strategy, for a given video stream that can be split into flows sent on different network paths between the streaming server, and the media client. The rate constraints are directly given by the network status, as shown before, and the overall streaming rate can be adapted by simple operations at the server (e.g., packet filtering). We can formulate the optimal multipath rate allocation problem as follows.

Given a network graph $G$, the optimization problem consists in jointly finding the optimal sending rate for a video packet stream, along with the optimal subset of network paths to be used for transmission, such that the end-to-end distortion is minimized. Equivalently, using the flow tree representation of the network graph proposed in Section II-B, the optimization problem translates into finding the optimal rate allocation for each of the flows in the tree, such that the video distortion is minimized. It can be formulated as follows.

Multimedia Rate Allocation Problem (MMR): Given the network graph $G$, the number of different paths or flows $n$, the video sequence characteristics $(\Gamma)$, and the total streaming 
budget $Q$, find the optimal rate allocation $\overrightarrow{R^{*}}=\left[r_{1}, \ldots r_{n}\right]^{*}$ that minimizes the distortion metric $D$ :

$$
\begin{aligned}
\overrightarrow{R^{*}} & =\underset{\vec{R}}{\arg \min } D\left(r_{1}, \ldots r_{n}\right) ; \\
& =\underset{\vec{R}}{\arg \min } f(R, \pi, \Gamma)
\end{aligned}
$$

where $R=\sum_{i=1}^{n} r_{i}$ and $\pi=\sum_{i=1}^{n} p_{i} \cdot r_{i} / \sum_{i=1}^{n} r_{i}$, under the following constraints.

1) Budget Constraints: $\sum_{i=1}^{n} c_{i} \leq Q$.

2) Single Flow Constraints.

3) Multiple Flow Constraints.

In the next section, we present a detailed analysis of a typical distortion model for video sequences. While the nonconvexity of the optimization metric will not allow for an easy solution by integration of the constraints into a Lagrangian formulation, our analysis eventually allows us to define a simple algorithm, able to find the optimal rate allocation with linear time complexity.

\section{Flow RATE AlLOCATION ANALYSIS}

\section{A. End-to-End Distortion Model}

We introduce in this section a quite generic distortion model, which is able to capture the influence of the average encoding rate on the source distortion, as well as the impact of losses on the channel distortion. Recall that our objective is to find the best flow rate allocation, on a multipath networks with known average statistics. Hence, we are looking for an average distortion model, which is able to estimate the video quality of service in a stationary regime.

In low to medium bit rate video streaming, it is commonly accepted that the source distortion is a decaying exponential function on the encoding rate, while the channel distortion is proportional to the number of lost packets (i.e., the packet loss probability, when the number of packet per frame is independent of the bit rate) [10]. Hence, we can explicitly formulate the Mean-Square Error distortion metric as:

$$
D=\alpha \cdot R^{\xi}+\beta \cdot \pi
$$

where $\alpha, \beta \in \Re^{+}$and $\xi \in[-1,0]$ are parameters that depend on the video sequence. This distortion model is a simple and general approximation that follows closely the behavior of more sophisticated distortion measures, such as those proposed in [11]-[13]. Since it is suitable for most common streaming strategies where the number of packets per frame is independent of the encoding rate, we use the model of (8) in the remainder of that paper. It can be noted that our simple model does not take into account the exact characteristics of the loss process, and that it mostly captures the effect of independent losses. We assume that bursts of losses on the video packet stream are quite unlikely due to the partitioning in multiple flows. Simple interleaving can also be applied to reduce the effects of bursts, if delay permits it. Finally, we should stress out that bursts of video packets losses are in general less penalizing for the channel distortion [14], so that our model has the advantage to provide a worst case estimate of the end-to-end distortion.

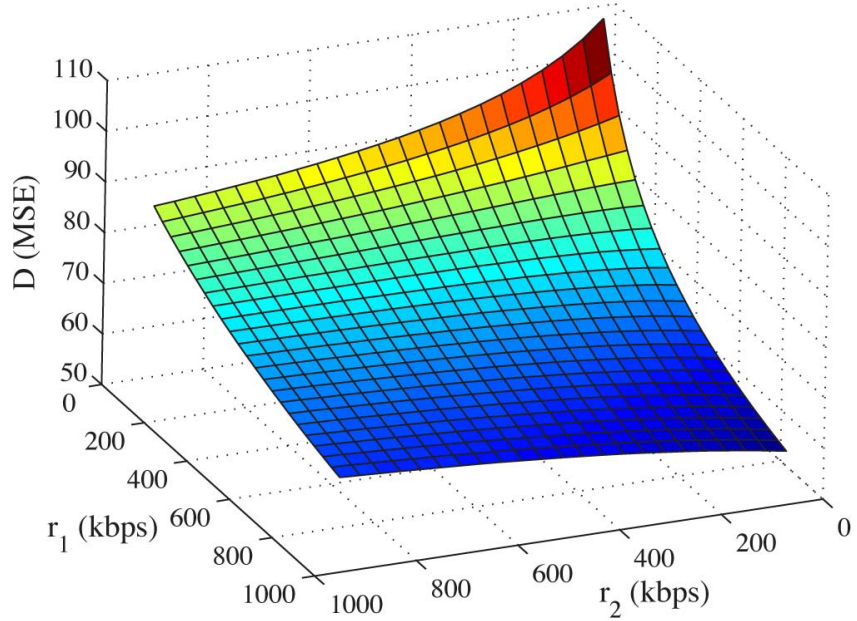

Fig. 3. Overall distortion measure for two network paths in function of available rates, $\alpha=1.76 \cdot 10^{5}, \xi=-0.658, \beta=1750, p_{1}=0.02$, and $p_{2}=0.04$.

Before going deeper in the analysis of flow rate allocation, we propose a simple example to illustrate the behavior of the end-to-end video distortion in a multipath scenario. We consider a basic network scenario consisting of two disjoint network paths, $P_{1}$ and $P_{2}$, with bandwidth $b_{1}=b_{2}=1000 \mathrm{kbps}$, and loss probabilities $p_{1}=2 \%$ and $p_{2}=4 \%$, respectively. Consider two independent flows $\mathcal{F}_{1}$ and $\mathcal{F}_{2}$ composing the same video stream, and traversing the two network paths with streaming rates $r_{1} \leq b_{1}$, and $r_{2} \leq b_{2}$. The evolution of the distortion function given in (8) is presented in Fig. 3, for a test video sequence (i.e., Foreman CIF).

As expected, we observe that the decrease in distortion is larger if we increase the rate of flow $\mathcal{F}_{1}$, than if we equivalently increase the rate of flow $\mathcal{F}_{2}$. This behavior is due to the lower loss probability that affects the path followed by the flow $\mathcal{F}_{1}$. In the same time, we observe that the distortion metric is always decreasing with the increase of $r_{1}$, hence it is optimal to fully utilize the bandwidth of the path with the smallest loss probability. In this case, for a given packet loss rate, it is better to increase the quality of each video frame by augmenting the rate $r_{1}$, as expected.

More interestingly, Fig. 4 shows that the behavior of the distortion as a function of the rate $r_{2}$, depends on the value of the rate $r_{1}$. For high values of $r_{1}$, the distortion can even increase with growing rate $r_{2}$. Beyond a given value of the streaming rate on the most reliable network path, adding an extra flow can degrade the end-to-end quality of the media application since the packet loss rate increases. In this case, the negative influence of the error process on the second network path is greater than the improvement brought by additional streaming rate. Such a behavior is the key to explain why using all the paths to their full bandwidth does not necessarily result in an efficient streaming strategy. Finally, the same type of behavior can be observed for stored video packet streams that are built on video packets, and error control packets (e.g., Forward Error Correction). In this case, the sensitivity of the channel distortion is obviously lower for low error rates, but rapidly increases when the channel protection becomes insufficient. 


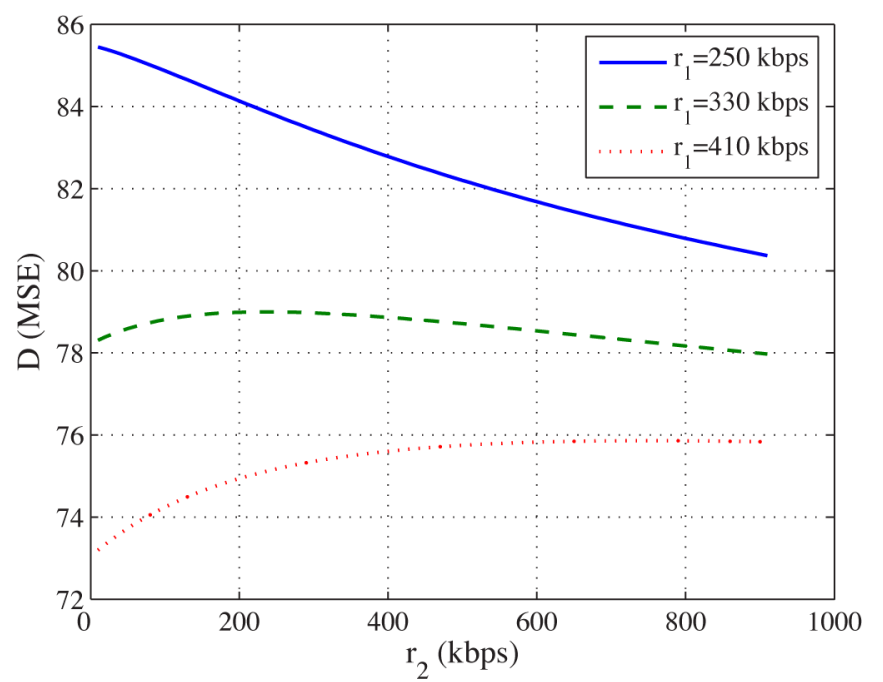

Fig. 4. Overall distortion behavior as a function of $r_{2}$, for various fixed values of $r_{1}$.

\section{B. Maximum or Null Flows}

We now generalize the previous observations, and derive theorems that guide the design of an optimal rate allocation strategy for a given video packet stream. This section shows that, in the optimal rate allocation, a flow is either used at its full bandwidth, or not used at all. Furthermore, the optimal rate allocation always chooses the lowest loss probability paths, i.e., a path shall not be selected, unless all other paths with a lower loss probability have been picked before. We start from an ideal streaming scenario with unlimited budget and disjoint network paths, and eventually add budget and flow constraints, which are however shown not to affect the initial findings.

Assume that the $n$ disjoint network paths are represented into a tree of flows as explained in Section II-B. Without loss of generality, we further assume that flows $\mathcal{F}_{i}$ with $1 \leq i \leq n$, are arranged in increasing order of the loss probability, i.e., $p_{1}<$ $p_{2}<\ldots<p_{n}$. We note that, from the distortion metric point of view, any two flows $\mathcal{F}_{i}$ and $\mathcal{F}_{j}$, with rates $r_{i}$ and $r_{j}$ and traversing paths $P_{i}$ and $P_{j}$ with the same loss probability $p_{i}=p_{j}$, can be observed as a single flow affected by the same loss probability $p_{i}$, and having an aggregated rate $r_{i}+r_{j}$. Under these generic settings, we first claim that the optimal rate allocation either uses a network path to its full bandwidth, or does not use it at all.

Theorem 1 (On-Off Flows): Given a flow tree with independent flows $\mathcal{F}_{i}$ having rates $r_{i} \in\left[0, b_{i}\right]$ and a distortion metric as defined in (8), the optimal solution of the MMR problem when all the paths are disjoint, lies at the margins of the value intervals for all $r_{i}$, i.e., the optimal value of $r_{i}$ is either 0 or $b_{i}$, $\forall i: 1 \leq i \leq n$.

Proof: Deriving the distortion $D$ given in (8), with respect to the rate $r_{i}, \forall i: 1 \leq i \leq n$, we obtain

$$
\begin{aligned}
\frac{\partial D\left(r_{1}, \ldots r_{n}\right)}{\partial r_{i}} & =\alpha \xi\left(\sum r_{i}\right)^{\xi-1}+\beta \cdot \frac{p_{i} \sum r_{j}-\sum p_{j} r_{j}}{\left(\sum r_{i}\right)^{2}} \\
& =\alpha \xi\left(\sum r_{i}\right)^{\xi-1}+\beta \cdot \frac{\sum_{j} r_{j} \cdot\left(p_{i}-p_{j}\right)}{\left(\sum r_{i}\right)^{2}}
\end{aligned}
$$

Observe that the condition for an extremum, $\partial D\left(r_{1}, \ldots r_{n}\right) / \partial r_{i}=0$ for any $r_{i}$, implies:

$$
\alpha \cdot \xi \cdot\left(r_{i}+\lambda\right)^{\xi+1}+\beta \cdot \mu=0
$$

where $\lambda$ and $\mu$ are constants independent on $r_{i}$. Since $0 \leq \xi+$ $1 \leq 1$, the equation has a single finite solution

$$
r_{i}^{*}=\sqrt[\xi+1]{\frac{\beta \cdot \mu}{-\alpha \cdot \xi}}-\lambda
$$

In the same time, the derivative in any point $r_{i}<r_{i}^{*}$ is positive, while to the right of the optimal value, it is negative (since $\xi \in[-1,0)$, and all other terms are positive). Hence, $r_{i}^{*}$ is a point of local maximum for the distortion function $D$, which means that only values at the margins of the value interval for $r_{i}$ can minimize the objective function. ${ }^{1}$

It can be further observed that, in the case of $r_{1}, \partial D / \partial r_{1}<0$, for any positive value of $r_{1}$ (since $\xi<0, \alpha, \beta>0$ and $p_{1}-p_{j}<$ $0, \forall j: 2 \leq j \leq n$ ). Hence the value $r_{1}=b_{1}$ always minimizes the objective function, and is part of the optimal solution.

Corollary 1: Given a flow tree with independent flows $\mathcal{F}_{i}$ having rates $r_{i} \in\left[0, b_{i}\right]$ and a distortion metric as defined in (8), the optimal solution of the MMR problem when all paths are disjoint, allocates $r_{1}=b_{1}$, where the path $P_{1}$ is the path with the lowest loss probability.

Theorem 1 greatly reduces the search space for an optimal solution to the MMR optimization problem. Hence we can rewrite the optimal streaming solution as a vector $\Phi$ of boolean values $\phi_{i}$ for each flow $\mathcal{F}_{i}$, where $\phi_{i}=1$ means that path $P_{i}$ is used with full rate $r_{i}=b_{i}$, and $\phi_{i}=0$ denotes the fact that the path $P_{i}$ is not used by the streaming application. The previous corollary further says that $\Phi=\left[\phi_{1}=1, \phi_{2}, \ldots, \phi_{n}\right]$ is part of the optimal solution.

For bounded intervals for all rates $r_{i}, 2^{n-1}$ computations are sufficient for finding the optimal solution vector. For practical scenarios, with a limited number of available network paths between a server and a client, this number of computations is in general quite low. We can however further constrain the search space by considering that the optimal rate allocation always uses first the network paths with the smallest loss probabilities.

Theorem 2 (Parameter Decoupling): Given a flow tree with independent flows $\mathcal{F}_{i}$ having rates $r_{i} \in\left[0, b_{i}\right]$ and a distortion metric as defined in (8), the structure of the optimal rate allocation is $\Phi^{*}=[1,1, \ldots, 1,0,0, \ldots 0]$.

Proof: We prove the result by induction. Recall that the network paths/flows are arranged in increasing order of their loss probabilities $p_{i}$. We have already seen that $\Phi=\left[\phi_{1}=\right.$ $\left.1, \phi_{2}, \ldots, \phi_{n}\right]$ is part of the optimal solution. Next we show that, for $n \geq 3, \Phi=\left[\phi_{1}=1, \phi_{2}=0, \phi_{3}=1, \phi_{4}, \ldots, \phi_{n}\right]$ cannot be part of the optimal solution.

For the sake of clarity, let us remove $\phi_{i}$ 's with $i>3$ from the notation, since they stay constant in our proof. By contradiction, assume that $\Phi$ is part of the optimal solution. It means that $D\left(b_{1}, 0, b_{3}\right)<D\left(b_{1}, 0,0\right)$. Since the paths are ordered with increasing values of the loss probabilities and considered to be disjoint, we can always transfer part of the rate from $\mathcal{F}_{3}$

${ }^{1}$ Since $r_{i}^{*}$ is the only finite solution, this statement is valid even if $r_{i}^{*}$ is not contained in $\left[0, b_{i}\right]$. 
to $\mathcal{F}_{2}$, and improve the distortion. Let $r_{2}=\min \left(b_{2}, b_{3}\right)$, and $r_{3}=\left[b_{3}-b_{2}\right]^{+}$. We have

$$
D\left(b_{1}, r_{2}, r_{3}\right)<D\left(b_{1}, 0, b_{3}\right)<D\left(b_{1}, 0,0\right) .
$$

The first inequality comes from the definition of the distortion metric, the second one from the assumption that $\Phi$ is part of the optimal solution. We can further distinguish two cases.

- $b_{2} \leq b_{3}$. Then, $r_{2}=b_{2}$, and $r_{3} \geq 0$ and, according to Theorem 1, there exists a solution $D\left(b_{1}, b_{2}, b_{3} \cdot \phi_{3}^{*}\right)<$ $D\left(b_{1}, b_{2}, r_{3}\right)<D\left(b_{1}, 0, b_{3}\right)$, with $\phi_{3}^{*} \in\{0,1\}$. $\Phi$ cannot be part of the optimal solution since $\phi_{2}^{*}=1$, which contradicts our assumption.

- $b_{2}>b_{3}$. Then, $r_{2}=b_{3}$ and $r_{3}=0$, and we have $D\left(b_{1}, b_{3}, 0\right)<D\left(b_{1}, 0, b_{3}\right)<D\left(b_{1}, 0,0\right)$. From Theorem 1 , there exists an even better solution where $r_{2}=b_{2}$, leading to $\Phi^{*}=[110]$, which again contradicts our assumption.

Next, we prove that $\Phi=\left[1 \ldots 1,0 \ldots 0,1 \ldots 1, \phi_{m}, \ldots, \phi_{n}\right]$ cannot be part of the optimal solution. In other words, we prove that the optimal rate allocation $\Phi^{*}$ can only be a series of consecutive 1's, followed by a series of consecutive 0's. Let $\phi_{j}=0$ and $\phi_{k}=0$, with $j, k<m$, be the start and end of the series of consecutive 0 's in $\Phi$. Following the same reasoning as before, transferring rate from flows $\mathcal{F}_{i}$, with $k+1<i<m-1$, to $\mathcal{F}_{j}$ can only improve the overall distortion. If $b_{j} \leq \sum_{i=k+1}^{m-1} b_{i}$, it directly leads to a solution with $\phi_{j}=1$ that is better than $\Phi$. Otherwise, it leads to a solution where $r_{j}=\sum_{i=k+1}^{m-1} b_{i}$ and $\phi_{i}=0$ for $j<i<m$, which can further be improved by choosing either $r_{j}=b_{j}$ or $r_{j}=0$ (from Theorem 1). Both cases exclude $\phi_{j}=0$ and $\phi_{i}=1$ for $j<i<m$ to be simultaneously part of the optimal solution. The proof can further be extended to the complete series of consecutive 0's in $\Phi$.

The previous theorems show that we can find the optimal solution for our optimization problem by iteratively searching all available network paths $P_{i}$, taken in ascending order of their loss probability $p_{i}$. Once we find a network path that can improve the overall distortion result, before using it, we have to make sure that all other network paths with better loss parameters are already used to their maximum available bandwidth. Hence, the search space is reduced to a maximum of $n$ computations.

\section{Nondisjoint Network Paths}

We now show that, relaxing the assumption on disjoint network paths in the original network graph does not change the general form of the optimal solution. We assume that in the original network graph $G$, there is at least one bottleneck link $L_{u}$, shared by at least two distinct network paths. Let $\mathcal{B}_{u}=\left\{P_{k}\right\}$, $\forall k: L_{u} \in P_{k}$, be the set of paths sharing the bottleneck link $L_{u}$. In this particular case, while using any of the paths $P_{k}$ alone yields an available bandwidth $b_{k} \leq \rho_{u}$, using all of them in the same time results in an aggregated bandwidth $\sum_{k} b_{k} \geq \rho_{u}$. Note that $L_{u}$ may, or may not be a bottleneck link for any of the paths $P_{k}$, treated independently. The paths $P_{k}$ in $\mathcal{B}_{u}$ are called "joint paths" The following theorem regulates the sharing of bandwidth $\rho_{u}$ among paths $P_{k}$ :

Theorem 3 (Bottleneck Bandwidth Sharing): Let $L_{u}$ be a bottleneck link for the set of paths $\mathcal{B}_{u}=\left\{P_{k}\right\}$ in $G$, the bottleneck link bandwidth $\rho_{u}$ shall be shared among paths $P_{k}$ in a greedy way, starting with the path affected by the lowest loss probability.

Proof: As previously, let the paths $P_{k} \in \mathcal{B}_{u}$ be arranged in increasing order of their loss probabilities $p_{k}$. Let further $\vec{R}_{u}=\left\{r_{k}\right\}_{P_{k} \in \mathcal{B}_{u}}$ denote a valid rate allocation among the nondisjoint paths. Recall that a valid rate allocation has to satisfy the single flow constraints (i.e., $r_{k} \leq b_{k}, \forall k$ ), and the multiple flow constraints, $\sum_{k} r_{k} \leq \rho_{u}$. Let $P_{i}$ be the path with the lowest loss probability in $\mathcal{B}_{u}$. If $r_{i}<b_{i}$ in $\vec{R}_{u}$, and $\sum_{k, k \neq i} r_{i}>0$, one can always find a better rate allocation by transferring rate from other flows sharing the same bottleneck link, to the flow $\mathcal{F}_{i}$. Since the total rate stays constant, the rate transfer does not affect the source distortion, and does not violate the multiple flow constraints. It however reduces the channel distortion, resulting in improved overall performance. By induction, the proof can be extended to all the nondisjoint paths. This shows that for any valid, but nongreedy rate allocation $\vec{R}_{u}=\left\{r_{k}\right\}_{P_{k} \in \mathcal{B}_{u}}$, there exists a better solution that uses in priority the lowest loss probability paths.

Note that the previous theorem can easily be extended to any number of bottleneck links in $G(V, E)$, and to paths that belong to different sets $\mathcal{B}_{u}$ in the same time. Theorem 3 allows to extend Theorem 2 to generic network graphs, with potentially nondisjoint paths. It results in the general rule that paths should be taken in the increasing order of their loss probability, and that all the flows should be used to their maximum capacity, that can be limited by joint bottleneck links, before considering an additional flow. Interestingly, any network scenario can thus be transformed into a disjoint flow tree, by a greedy allocation of joint bottleneck bandwidths to flows affected by lower loss probabilities first. After this transformation, applying Theorem 1 and Theorem 2 will yield the optimal rate allocation for the given streaming scenario.

Finally, we can relax the assumption of independent flows in Theorem 1, by proper adaptation of the maximal bandwidth of all nondisjoint paths.

Corollary 2: Given a generic flow tree with flows $\mathcal{F}_{i}$ ordered in increasing order of their loss probability, and a distortion metric as defined in (8), the optimal solution of the MMR problem lies at the margins of the value intervals for all $r_{i}$, i.e., the optimal value of $r_{i}$, $\forall i: 1 \leq i \leq n$, is either 0 or $b_{i}^{\prime}=\min \left(b_{i}, w_{i}\right)$, where $w_{i}=\min _{u: L_{u} \in P_{i}}\left\{\rho_{u}-\sum_{k: L_{u} \in P_{k} \text { and } p_{k}<p_{i}} b_{k}^{\prime}\right\}$.

Finally, multipath streaming applications may also have to respect a budget constraint $Q=\sum_{i} k r_{i}$, or a maximal encoding rate $R_{c}$ in the case of pre-encoded media sequence. These constraints can be modeled as an additional virtual bottleneck link going out of the server. Fig. 5 shows such a transformation, where link $L_{0}$ and node $N_{0}$ are added to the topology in order to incorporate the previous overall constraints. Link $L_{0}$ should not influence the loss process of the intermediate network, hence $\theta_{0}=0$. The bandwidth $\rho_{0}$ is established at $\rho_{0}=\min \left((Q / k), R_{c}\right)$, where $Q$ and $R_{c}$ are simply set to $\infty$ in the case where there are no limitative factors on the total bandwidth. Applying Theorem 1, Theorem 2 and Theorem 3 on the new network graph $G^{\prime}=\left(E, V, L_{0}, N_{0}\right)$, yields an optimal rate allocation for a stored packet stream, which fully takes into account the budget and encoding rate constraints. 


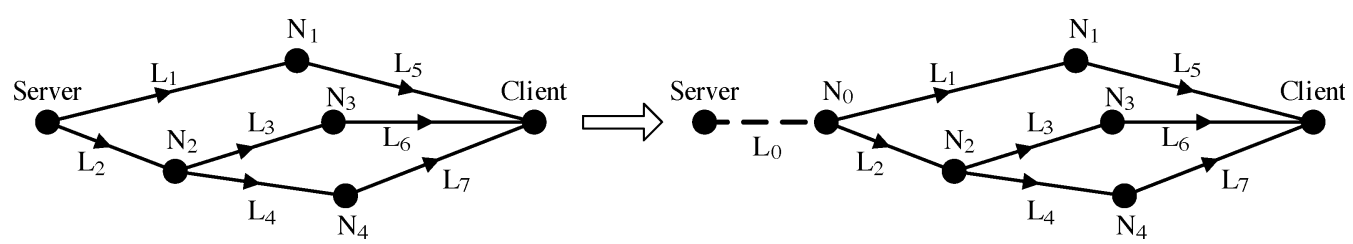

Fig. 5. Inclusion of budget or encoding rate constraints as a virtual network link in the original network graph.

\section{Rate Allocation Algorithm}

\section{A. Linear Complexity Search Algorithm}

The analysis proposed in Section III shows that a simple algorithm can find the optimal rate allocation by parsing all available network paths in ascending order of their loss probability. Denote $\Phi_{i}=\left[\phi_{1}, \ldots, \phi_{n}\right]$ a solution vector with $\phi_{j}=1, \forall j \leq i$, and $\phi_{j}=0$ otherwise. $R\left(\Phi_{i}\right)=\sum_{j=1}^{i} r_{j}$ becomes the cumulative rate of the first $i$ flows, whose individual rates have been chosen according to Corollary 2 . The overall loss probability of the first $i$ flows, $\pi\left(\Phi_{i}\right)$, is then given by $\pi\left(\Phi_{i}\right)=$ $\sum_{j=1}^{i} p_{j} \cdot r_{j} / \sum_{j=1}^{i} r_{j}$. The Search Algorithm iteratively computes $D\left(R\left(\Phi_{i}\right), \pi\left(\Phi_{i}\right)\right)$, for $1 \leq i \leq n$, and the optimal rate allocation is the policy $\Phi^{*}$ that minimizes the distortion metric

$$
\Phi^{*}=\underset{\Phi_{i}, 1 \leq i \leq n}{\arg \min } D\left(R\left(\Phi_{i}\right), \pi\left(\Phi_{i}\right)\right) .
$$

The algorithm will be able to find the global optimal rate allocation only after parsing all available network paths. From the previous theorems, the optimal rate allocation solution $\Phi^{*}$ takes the form of a consecutive series of 1's, followed by a consecutive series of 0 's, hence requiring a maximum of $n$ computations. We propose below a few conditions for early termination, which may avoid to test all possible solutions, while still ensuring a global optimal solution. These conditions represent an extra complexity reduction of the optimum search. ${ }^{2}$

\section{B. Conditions for Early Termination}

The search algorithm has to iteratively compute $D\left(\Phi_{i}\right)$, for increasing values of $i$. A full search through $n$ possible solutions may however be avoided, if any one of the following termination conditions is verified.

1) Distortion Limitation: If $D\left(\Phi_{i-1}\right) \leq \beta \cdot p_{i}$, then the optimal rate allocation contains $\phi_{j}=0, \forall j \geq i$. It can be shown from the distortion function given in (8) that $\lim _{b_{i} \rightarrow \infty} D\left(\Phi_{i}\right)=\beta \cdot p_{i}$, when other rates $b_{j}$ stay unchanged, $b_{i} \rightarrow \infty$
$\forall j \neq i$. Hence, for a value of $D\left(\Phi_{i-1}\right) \leq \beta \cdot p_{i}$, adding another flow on path $P_{i}$ will asymptotically increase the overall distortion metric to $\beta \cdot p_{i}$. Therefore, for any positive value of $b_{j}$, with $j \geq i$, and $p_{j} \geq p_{i}$, adding extra rate on path $P_{j}$ will only increase the distortion measure in this case.

${ }^{2}$ Please note that the problem in general can be solved in less than linear time (e.g., $O(\log (n))$ computations). However, due to the limited number of paths chosen for transmission, as reflected by our simulation results, the linear time algorithm that parses the available network paths in ascending order of their loss probability, along with the conditions for early termination, achieve the optimal solution even faster.
2) Path Bandwidth Limitation: Solving the equation $D\left(\Phi_{i-1}\right)=D\left(\Phi_{i}\right)$ for the variable $r_{i}$ may provide, except the trivial solution $r_{i}=0$, another positive, finite value for $r_{i}$, noted as $r_{i}^{\prime}$. This second solution happens in the case where $D\left(\Phi_{i-1}\right) \geq \beta \cdot p_{i}$ and $R\left(\Phi_{i-1}\right) \geq e^{\left(\left(\ln -(\beta / \alpha \cdot \xi)\left(p_{i}-\pi\left(\Phi_{i-1}\right)\right)\right) / \xi\right)}$. The later value is obtained by solving $\partial D\left(\Phi_{i}\right) /\left.\partial r_{i}\right|_{r_{i}=0}=0$. It represents the minimum rate $r_{i-1}$, after which, adding an extra rate $r_{i}$ could lead to an increase in distortion. In the case where $b_{j} \leq r_{i}^{\prime}, \forall P_{j}$ with $j \geq i$, adding another flow, will not decrease the overall distortion, since unused bandwidth is not sufficient anymore to compensate for the increase in loss probability in case an extra flow is added. In that case, according to Theorem 2 and to the definition of the distortion metric, $D\left(\Phi_{j}\right) \geq D\left(\Phi_{i-1}, r_{i}^{\prime}\right)$, hence $D\left(\Phi_{j}\right) \geq D\left(\Phi_{i-1}\right), \forall j \geq i$.

Any of the above criteria represents a sufficient condition for search termination from the theoretical point of view, and can be applied at any stage of the optimal solution computation.

\section{Rate Allocation Algorithm}

This section presents a simple algorithm that computes the optimal rate allocation for the optimization problem. The previous theorems and conditions for termination represent the keys for a fast search through the flow tree. Assume that the server knows, or can predict the parameters of the intermediate network links, and the sequence-dependent distortion parameters. Initially, the network graph is transformed into a tree of flows $\mathcal{F}_{i}$, sorted along increasing values of the loss probabilities $p_{i}$, with greedy assignment of joint bottleneck link bandwidths. In case where two network paths have the same end-to-end loss probability, they are considered as a single path with aggregated bandwidth. The search for an optimal solution of the shape given by Theorem 2 is performed iteratively. At each step, the early termination conditions are verified. Once any of them is satisfied, or when the algorithm finishes the search of all flows, the algorithm stops and outputs the optimal multipath rate allocation strategy. Algorithm 1 proposes a sketch of the rate allocation algorithm.

During the initialization process, Algorithm 1 must compute all available paths between the streaming server $S$ and the client $C$. This is a well-known problem in graph theory, and a solution can be easily found by implementing a depth-first search $(D F S)$ [15], for example. The algorithm then arranges the discovered network paths as a flow tree in ascending order of their end-to-end loss probabilities. Any sorting algorithm of complexity $O(n \log (n))$ can be used. After the flow tree is constructed, the core of the algorithm finds the optimal rate allocation with a complexity $O(n)$, at maximum. 


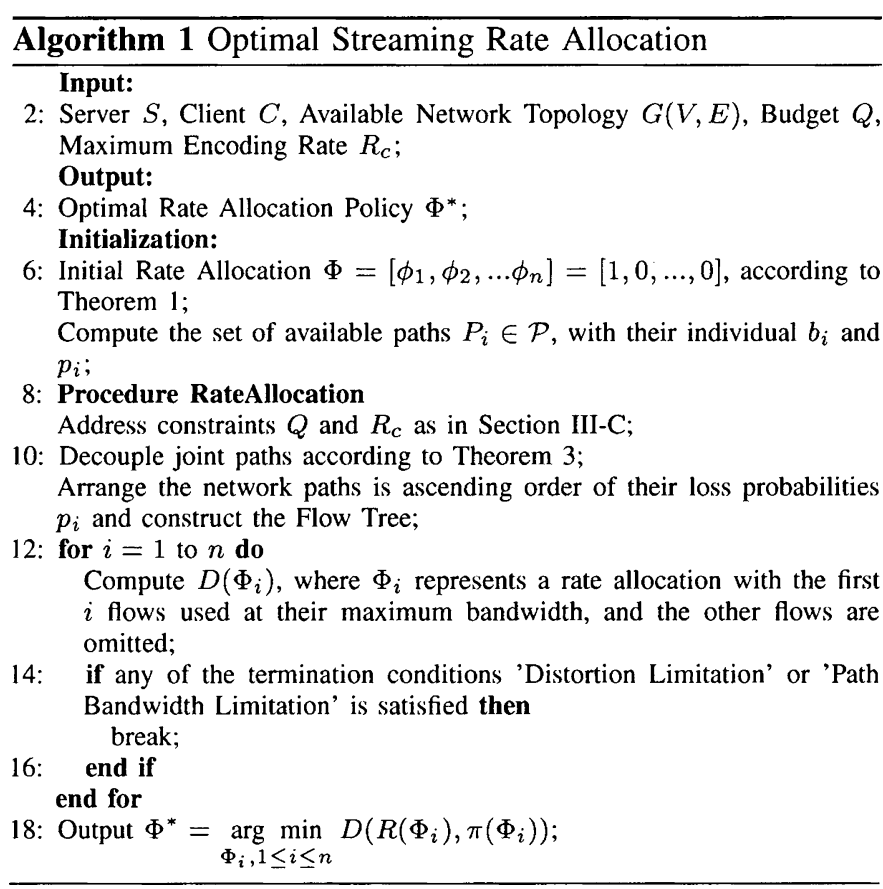

\section{Discussion}

In this section we discuss the practical deployment of the mechanisms proposed above, and some of its limitations. The problem formulation and the methodology for the optimal flow rate allocation of a given video packet stream over multipath networks are valid for numerous encoding scenarios, including off-line joint source and channel coding of media streams. We assume that the server is not able to perform complex coding operations in real-time, mostly for computational complexity and scalability issues. In such a scenario, adaptive streaming strategy mostly consist in finding the best routing strategy, and overall rate allocation, for the transmission of a given packet stream on a given multipath network. Additional benefits are offered when several versions of the same stream are available at the server. Due to the low complexity of our algorithm, the server could identify both the best transmission strategy, and the best stream to be sent, with an additional complexity that is only linear with the number of stored versions. Such a design choice is also beneficial in broadcast applications, where several clients are accessing the same stream. In such situations, fine adaptation of the packet stream to each individual client is impossible. Coupled with efficient packet partitioning strategy, our flow rate allocation solution however offers interesting perspectives in these scenarios.

In typical network infrastructures, bandwidth and loss rate are quite dynamic. However, they usually exhibit stable statistics on medium range timescales (i.e., in the order of few hundreds of milliseconds, to seconds). We assume that the server can estimate the average end-to-end bandwidth $r_{i}$ and loss probability $p_{i}$ of the available paths to the client, for such timeframes. Additionally, we assume that each path is characterized by a total end-to-end delay $\delta_{i}$, imposed on all packets traversing that path. Finally, the client imposes a maximum tolerable payback delay $\Delta$, after which it starts playing the media file. Given the estimated parameters $r_{i}, p_{i}$ and $\delta_{i}$, the server chooses the optimal transmission strategy in order to maximize the received media quality. While the fastest estimation mechanisms on end-to-end scenarios provide accurate results on time frames of a few seconds [16], our rate allocation mechanism converges to the optimal solution in a very small number of computations. Since our algorithm has a low complexity, it can be run periodically, with updated network parameter estimates. It ensures the best transmission strategy for a stored video stream, given the accuracy of the periodic network parameter estimation.

We identify a few typical scenarios where optimal rate allocation between multiple stream paths can bring interesting benefits in terms of media quality. In each of these examples, the application of the algorithm proposed above is straightforward.

1) Wired Overlay Network Scenarios (e.g., Content Distribution Networks). The media information from a server is forwarded towards the client by multiple servers belonging to the same overlay network. The client consumes the aggregated media from multiple network paths, and the algorithm proposed above can be applied directly to find the optimal rate allocation.

2) Wireless Network Scenarios (e.g., WiFi Networks). A wireless client can aggregate the media information transmitted on multiple wireless channels. Interference among transmission channels can be minimized by choosing nonoverlapping wireless channels (e.g., there are 8 nonoverlapping channels according to the IEEE 802.11a standard specifications), and by optimizing the transmission schedule in the wireless network [17]. The authors of [18] test a protocol stack that allows one wireless network card to be simultaneously connected to, and switch between, multiple networks in a transparent way for the application. In the same time, the authors of [19] present a video system over WLANs that uses multiple antennas in order to aggregate the rate of multiple wireless channels.

3) Hybrid Network Scenarios (e.g., UMTS/GPRS/WiFi Networks). A mobile client can simultaneously benefit from multiple wireless services in order to retrieve the media information from a server connected to the Internet backbone. Existing commercial products [20] can already maintain connectivity to multiple wireless services (e.g., UMTS, EDGE/GPRS and WiFi hotspots), and transparently switch at any time to the service that offers the best channel performance, for a fixed subscription price. It is only a question of time before such commercial products will be able to aggregate the resources of multiple such services in order to enhance the user streaming experience, and telecommunications operators are actively working on such systems.

All these applications can be modeled according to Section II-A, and the implementation of the proposed algorithm is generic and independent of any particular bandwidth and loss model, as long as the media flows can be considered independent in terms of losses. This assumption is valid in any disjoint path network scenario, since the media flows are independent in terms of both rate and losses. In generic network scenarios, our analysis still holds (namely the transformation between the network graph and the tree of flows in Section II-B), as long as the predominant losses affecting the 


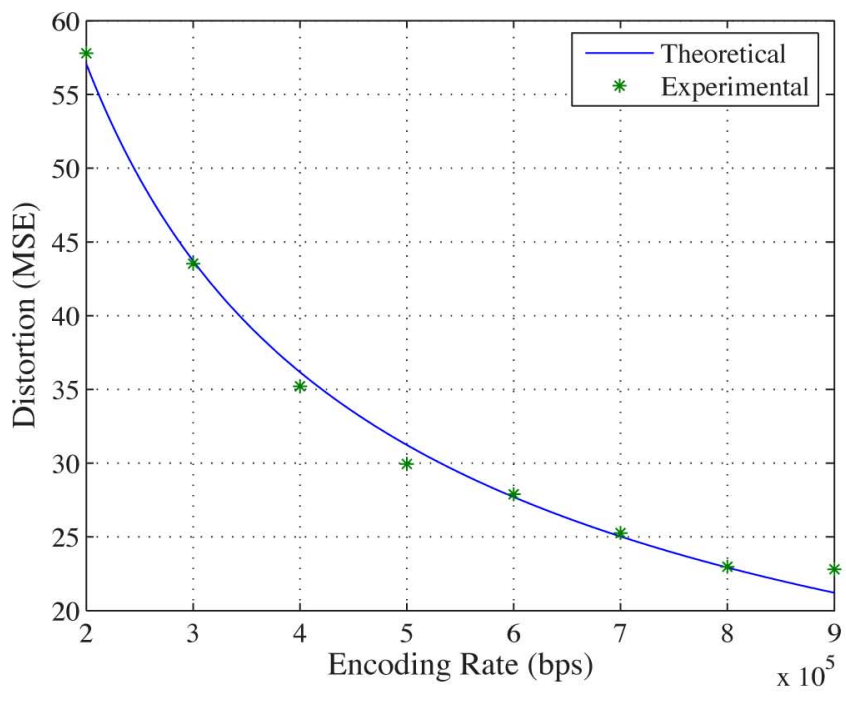

(a)

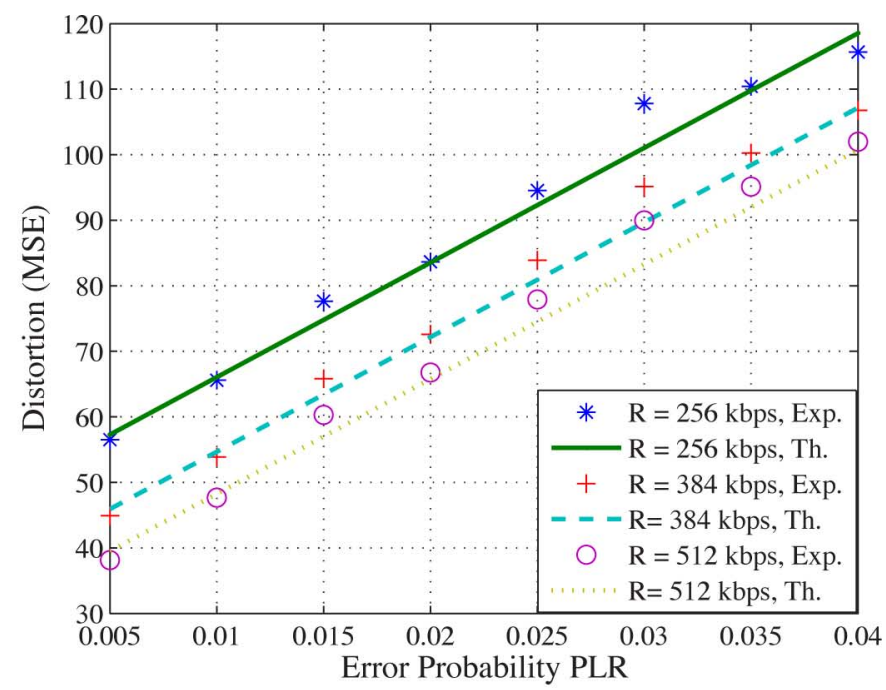

(b)

Fig. 6. Distortion model validation with video streaming experiments using the H264 encoder. (a) Encoding rate distortion validation and (b) loss distortion validation.

transmission process are independent among media flows (e.g., scenarios 2 and 3). An analysis of the rate allocation problem in general networks characterized by a Gilbert loss model (where the transformation in Section II-B can only be considered as an approximation) can be found in [10].

It can be noted that the applications mentioned above present in general a limited number of available network paths between the streaming server and the client. It is fairly easy for a server to continuously monitor these paths and to estimate their parameters. Based on these parameters, the execution of the proposed algorithm will output the optimal choice of paths and rates in terms of average media quality at the client. For very large network scenarios, it can be noted that the assumption of full knowledge about the network can be relaxed in setting up a distributed version of the proposed algorithm [21].

Finally, the network path selection and flow rate allocation problem does not consider media packetization and network scheduling issues. These issues are typically addressed at a lower and finer level. The packetized media stream can be split into packet flows corresponding to the chosen network paths, assuming a very simple scheduling algorithm. Given the estimated rates and delays on all the network paths, the server adapts the streaming rate to the available network bandwidth by simple operations on stored video packet stream. Then, it schedules the packets on the different paths according to the estimated arrival times at the client [22]. Network estimation errors and jitter can further be compensated at the client with the use of application dedicated buffers and conservative playback delay. Interleaving may also be implemented to fight against bursty loss processes when delays permits it.

\section{Simulation Results}

\section{A. Simulation Setup}

We test our optimal rate allocation algorithm in different network scenarios, and we compare its performance to heuristic rate allocation algorithms. We use an H.264 encoder, and the decoder implements a simple frame repetition error concealment strategy in case of packet loss. We concatenate the foreman_cif sequence to produce a 3000 frame-long video stream, encoded at 30 frames/s. The encoded bitstream is packetized into a sequence of network packets, each packet containing information related to one video frame. The packets are sent through the network on the chosen paths, in a FIFO order, following a simple earliest-transmission-time-first scheduling algorithm. We further consider a typical video-on-demand $\left(V_{O D}\right)$ streaming scenario, where the admissible playback delay is large enough (i.e., larger than the time required to transmit the biggest packet on the lowest bandwidth path). Hence, a video packet is correctly decoded at the client, unless it is lost during transmission due to the errors on the network links.

Our simulations first validate the distortion metric proposed in (8). Then, the performance of our optimal rate allocation algorithm is compared to heuristic rate allocation algorithms, on a set of random network topologies. Finally, we carefully analyze the behavior of optimal rate allocation for a particular network scenario, and discuss optimal solutions.

\section{B. Distortion Model Validation}

The video sequence is encoded at rates between $200 \mathrm{kbps}$ and $1 \mathrm{Mbps}$, and the mean-square-error $(M S E)$ between the original sequence and the decoded one is computed, in errorfree scenarios. Simulation results are compared in Fig. 6(a) to the distortion model values, whose parameters have been set to $\alpha=1.7674 \cdot 10^{5}, \xi=-0.65848$, and $\beta=1750$, respectively. We observe that the model distortion curve closely follows the experimental data, which validates the source distortion model.

In order to validate the loss distortion component $D_{L}$, random errors are introduced during the network transmission process, where each packet is lost with an independent loss probability $P L R$. Simulations are performed with different values of loss probabilities, and different encoding rates. We observe in Fig. 6(b) that the theoretical model closely approximates the experimental data, where each experimental point 


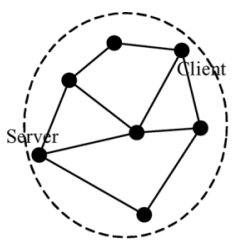

(a)

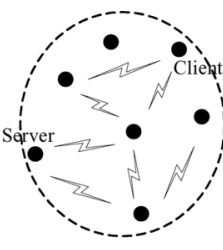

(b)

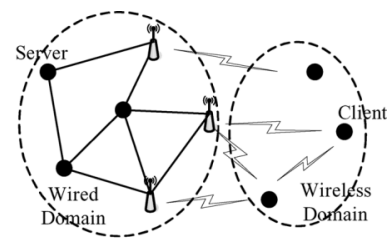

(c)
Fig. 7. Three network scenarios. (a) Wired network; (b) wireless network; and (c) hybrid network.

TABLE I

PARAMETERS FOR RANDOM GRAPH GENERATION

\begin{tabular}{|c|c|c|}
\hline Parameter & Wired Scenario & Wireless Scenario \\
\hline \hline Nr. of Nodes & 10 & 10 \\
\hline Connectivity Probability $\gamma$ & 0.4 & 0.6 \\
\hline$\rho_{\min }$ & $10^{6} \mathrm{bps}$ & $10^{5} \mathrm{bps}$ \\
\hline$\rho_{\max }$ & $3 \cdot 10^{6} \mathrm{bps}$ & $7 \cdot 10^{5} \mathrm{bps}$ \\
\hline$\theta_{\min }$ & $10^{-4}$ & $10^{-3}$ \\
\hline$\theta_{\max }$ & $5 \cdot 10^{-3}$ & $4 \cdot 10^{-2}$ \\
\hline \multicolumn{2}{|r}{} \\
\hline
\end{tabular}

is averaged over ten simulation runs. Even if it stays quite simple, the distortion model used in our work closely fits the average behavior of lossy video streaming scenarios. Note that the sequence-dependent parameters may obviously have different values for other encoders or other video sequences. The evolution of the distortion function however stays the same, independently of the exact values of these parameters.

\section{Rate Allocation Performance}

We now present the performance of the proposed optimal rate allocation algorithm, in various random network scenarios. We simulate three different categories of network topologies.

1) Wired network graphs, in which the edges between nodes are characterized by high bandwidth and low error probability.

2) Wireless network graphs, with low bandwidth and high error probability for the intermediate links.

3) Hybrid network scenarios, where the server is connected to the wired infrastructure, and the client can access the Internet via multiple wireless links.

The network scenarios are presented in Fig. 7. In each of the three cases, we generate 500 random graphs, where any two nodes are directly connected with a probability $\gamma$. The parameters for each edge are randomly chosen according to a normal distribution, in the interval $\left[\rho_{\min }, \rho_{\max }\right]$, for the bandwidth, and, respectively, $\left[\theta_{\min }, \theta_{\max }\right]$ for the loss probability. The parameters for the wired and wireless scenarios are presented in Table I. The hybrid scenario uses the parameters of both scenarios.

For each of the three types of scenarios, we compute the average end-to-end distortion when rates are optimally allocated, and we compare it to the results obtained by other simple rate allocation algorithms, namely, i) a single path transmission scenario, which selects the best path in terms of loss probability $\left(D_{P L R}\right)$, ii) a single path transmission scenario $\left(D_{R}\right)$, which
TABLE II

Average Distortion Results ( $M S E$ )

\begin{tabular}{|c|c|c|c|c|c|}
\hline Scenario & $D_{\text {opt }}$ & $D_{P L R}$ & $D_{R}$ & $D_{2 R}$ & $D_{M F}$ \\
\hline \hline Wireless & 91.2 & 99.74 & 122.861 & 143.79 & 108.52 \\
\hline Wired & 16.7 & 20.47 & 23.4 & 23.27 & 17.62 \\
\hline Hybrid & 63.4 & 73.809 & 83.97 & 92.533 & 72.57 \\
\hline
\end{tabular}

uses the best path in terms of effective bandwidth or "goodput" computed as $b_{i}\left(1-p_{i}\right)$, iii) a multipath transmission scenario $\left(D_{2 R}\right)$ that picks the best two paths in terms of goodput, and iv) a multipath transmission scenario that uses the maximum available number of flows, denoted as $D_{M F}$. The results, averaged over 500 random graphs are presented in Table II.

As expected, our algorithm provides the best average performance in the three considered scenarios. It has to be noted that, in each individual run of simulation, our algorithm never performs worse than any of the heuristic schemes. Also, we observe that, in the wireless scenario, the rate allocation that is the closest to the optimal strategy is the one offered by the use of the best single path in terms of loss rate. This can be explained by the high loss probabilities of the intermediate links, which cannot be compensated by extra rate added by subsequent flows. On the other hand, in the wired scenario, characterized by very small loss probabilities, the scheme that is the closest to the optimal solution is given by the greedy use of all available flows. In this case, the improvement brought by adding extra transmission rate outruns the losses suffered throughout the transmission process. The results for the hybrid scenario are situated, as expected, between the two extreme cases. The total streaming rates in the three scenarios are in average, $R=4 \mathrm{Mbps}$ for the wired scenario, $R=450 \mathrm{kbps}$ for the wireless scenario, and respectively $R=800 \mathrm{kbps}$ for the hybrid one.

Next, we study the benefit offered by optimal rate allocation, as compared to the simple heuristic schemes. The relevance of the optimal solution is measured by counting the number of simulation runs in which the optimal rate allocation brings an improvement of [0-5\%], [5-10\%], [10-20\%], and above $20 \%$, in terms of end-to-end video distortion, compared to the other streaming strategies. The results are presented in Figs. 8-10.

We observe that, in more than half of the cases, network flooding represents a good approximation of the optimal solution in the wired scenario where losses are rare. However, we argue that it is still worth applying the proposed rate allocation algorithm, because it is of very low complexity, and can still save network resources. In the wireless scenario, the best approximation is presented in most of the cases by the lowest loss probability path streaming. Still, in almost $40 \%$ of the simulation runs, the optimal rate allocation improves the distortion result by more than $10 \%$. Finally, in the hybrid scenario, the rate allocation algorithm provides significant quality improvements compared to all other heuristic approaches. It is also interesting to observe that the rate allocations based on the best goodput path, and best two goodput paths algorithms always provide the worst results.

We also compute the optimal average number of flows used in each simulation scenario, compared to the average number 


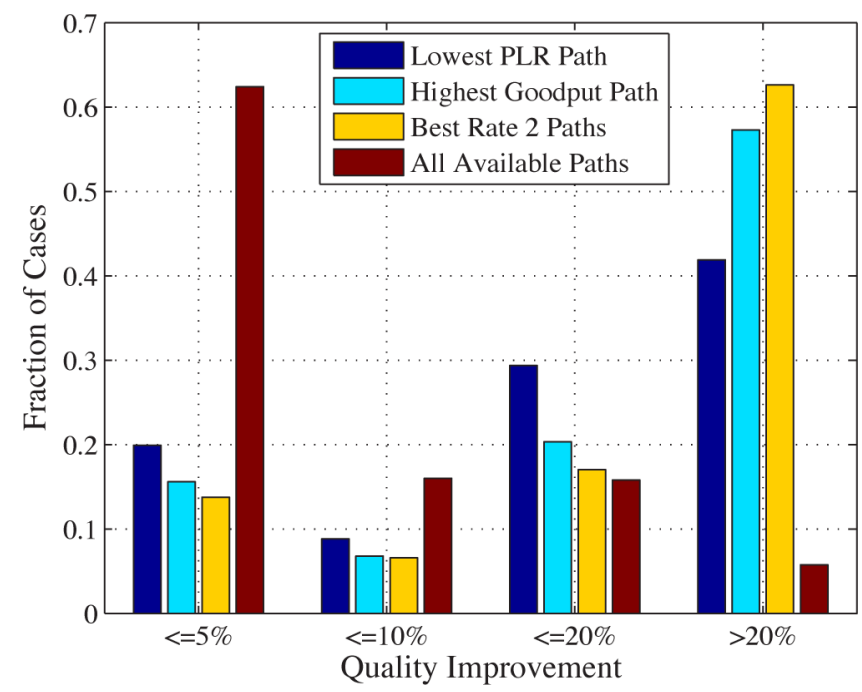

Fig. 8. Quality improvement versus heuristic rate allocation algorithms - wired scenario.

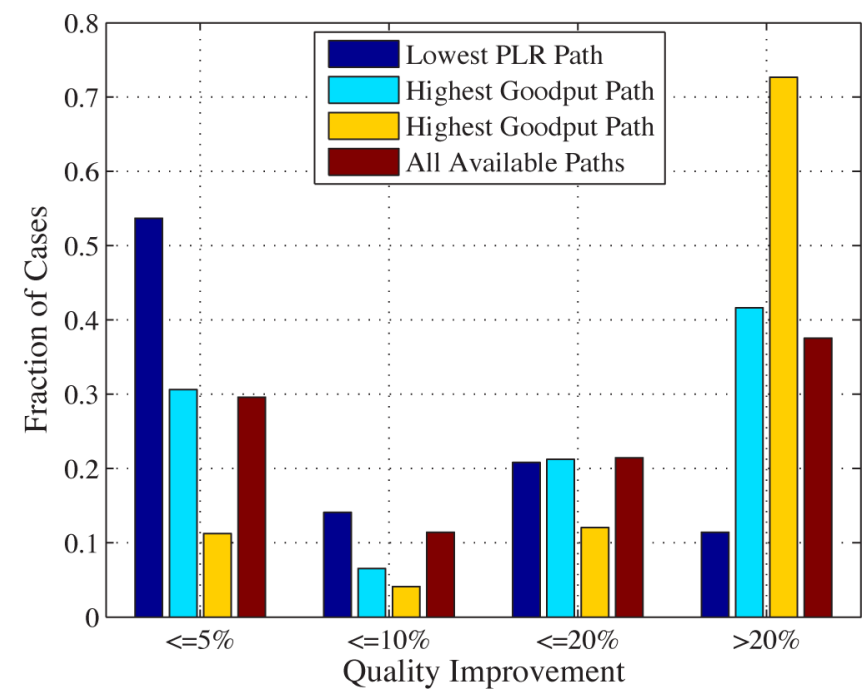

Fig. 9. Quality improvement versus heuristic rate allocation algorithms-wireless scenario.

of available paths. The results are presented in Table III. We observe that the wireless scenario uses the smallest number of flows, while the wired one has an average of no more than three flows, for a number of available paths that is far larger. From the multipath streaming point of view, it interestingly shows that, using a very large number of streaming paths does not contribute to an improvement of the video quality at the receiver. This is certainly interesting for the design of practical multipath streaming systems, where the number of paths that have to be synchronized, stays limited. The distribution of the number of flows used per simulation run, is presented in more details in Fig. 11.

In summary, we observe that a small number of transmission flows is sufficient for an optimal video quality at the receiver, in all simulation scenarios. Paths with lower error probability should be preferred to higher bandwidth paths in wireless scenarios, while in all-wired scenarios with low error probability, adding high-rate flows can improve the overall video quality. In

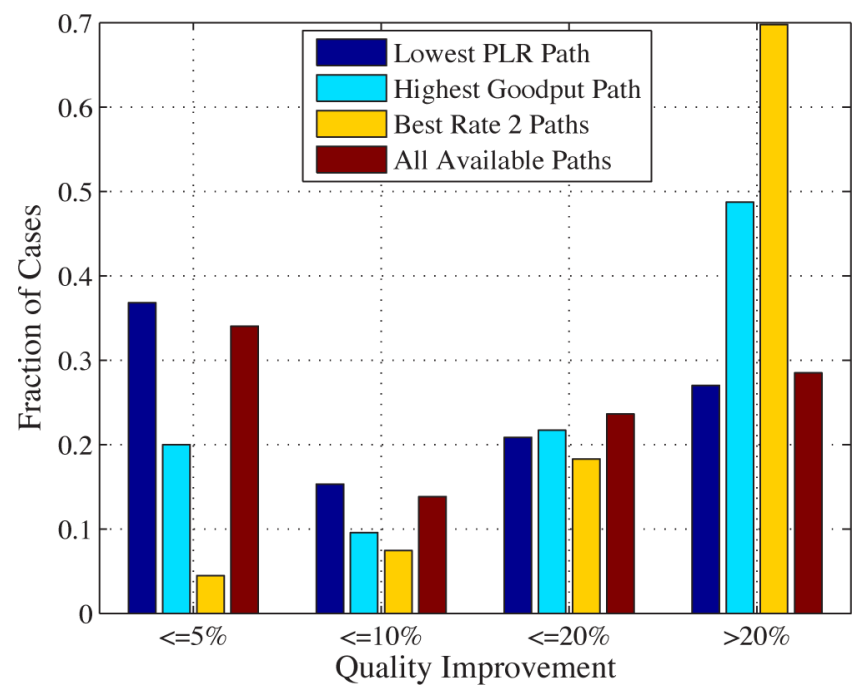

Fig. 10. Quality improvement versus heuristic rate allocation algorithms-hybrid scenario.

TABLE III

AVERAGe Number OF PATHS

\begin{tabular}{|c|c|c|}
\hline Scenario & Optimal Nr. & Available Nr. \\
\hline \hline Wireless & 2.04 & 5.04 \\
\hline Wired & 3.049 & 4.856 \\
\hline Hybrid & 2.17 & 4.419 \\
\hline
\end{tabular}

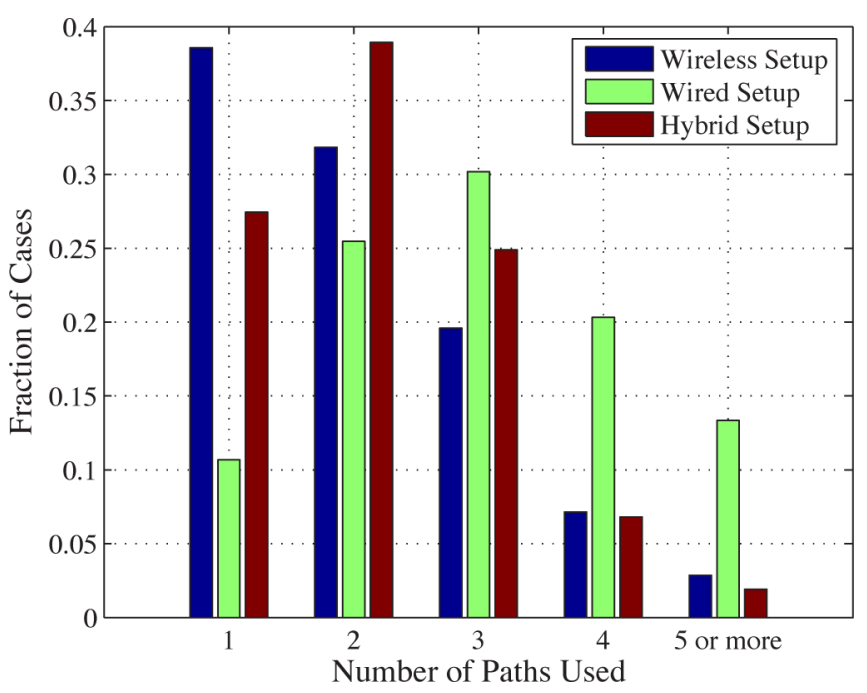

Fig. 11. Distribution of optimal number of paths for the 3 network scenarios.

hybrid scenarios, a compromise between the two tendencies is expected to provide the best end-to-end distortion.

\section{A Case Study}

This section proposes to analyze the performance of the optimal rate allocation algorithm in a given network scenario, illustrated in Fig. 12. The network parameters are presented in Table IV. For each of the five rate allocation algorithms, we compute the distortion measure according to the theoretical distortion metric, and we validate it against experimental values, 


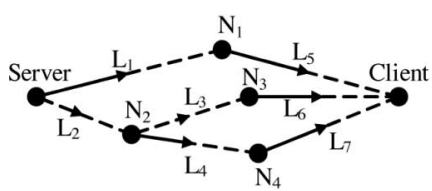

(a)

(c)

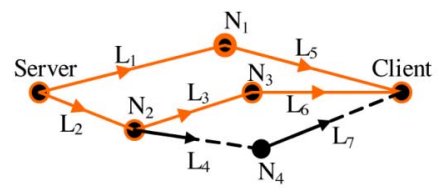

(e)

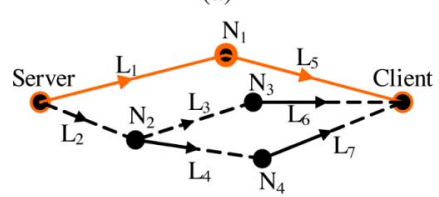

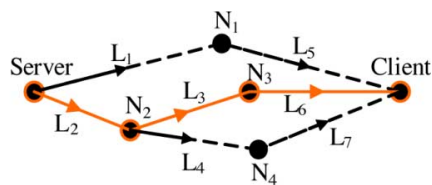

(b)

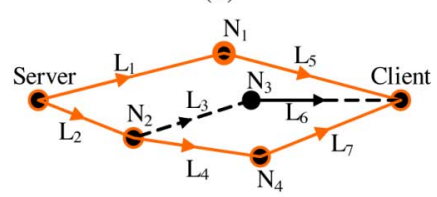

(d)

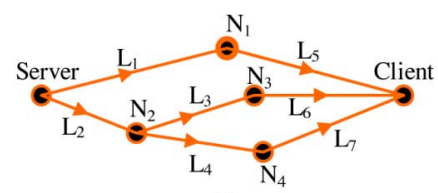

(f)
Fig. 12. One network topology example-optimal flow allocation and other heuristic algorithms. (a) Available network graph; (b)best PLR path; (c) best PLR path; (d) optimal flow allocation; (e) best goodput two paths; and (f) maximum flow graph.

TABLE IV

PARAMETER VALUES FOR THE LINKS IN $G(V, E)$

\begin{tabular}{|c|c|c|c|c|c|c|c|}
\hline Parameter & $L_{1}$ & $L_{2}$ & $L_{3}$ & $L_{4}$ & $L_{5}$ & $L_{6}$ & $L_{7}$ \\
\hline \hline$\theta_{i}$ & 0.02 & 0.01 & 0.035 & 0.01 & 0.015 & 0.035 & 0.01 \\
\hline$\rho_{u}(k b p s)$ & 256 & 384 & 256 & 128 & 256 & 256 & 128 \\
\hline
\end{tabular}

obtained from simulations with video sequences. Each experimental point is averaged over 10 simulation runs. Each video packet is scheduled on the network paths chosen by the given rate allocation algorithm, according to a simple first-available path first. In the same time, each video packet is affected by the individual loss process of each traversed network segment.

The $R$ and $\pi$ parameters, along with the model and experimental distortion values are presented in Fig. 13, for each of the algorithms. It can be observed that the optimal rate allocation algorithm outperforms all other heuristic-based strategies. The optimal rate allocation reaches a balance between total used bandwidth, number of network paths, and error probability that affects the streaming process. The example clearly shows that it is not optimal to use only the best paths in terms of rate. In the same time, the greedy use of all available network resources, does not provide better results. This clearly motivates the implementation of the proposed rate allocation algorithm, which optimizes the received video quality, without wasting network resources. Finally, it can be noted again that the theoretical distortion model represents a very good approximation of the experimental setup.

\section{RELATED WORK}

The research community has recently started to investigate the idea of multipath routing and streaming in order to improve the QoS of media applications. The authors of [23] present a distance-vector algorithm for finding multiple paths, while the authors of [24] present a multipath extension of Direct Source

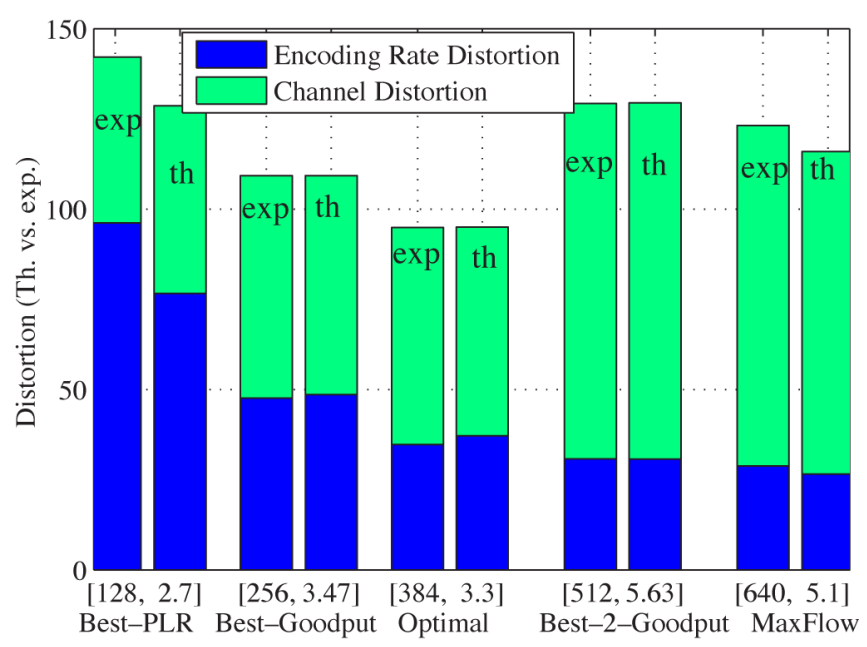

Rate Allocation Algorithm ([R (kbps), $\pi(\%)])$

Fig. 13. Network scenarios computation: theoretical distortion model versus experimentally computed distortion.

Routing for wireless ad-hoc environments. The purpose of the algorithms is to achieve load balancing over multiple paths, and to simultaneously minimize delays.

While all these works give a detailed analysis of the multipath routing problem form the networking point of view, we address the same problem from a media application perspective. The process of choosing the paths for transmission and their respective rate allocation is subordinated to achieving a better streaming experience, measured in terms of video distortion. The work presented in [25] addresses a similar problem of choosing the best path from a media perspective. However, the authors only address the question of path switching efficiency from the media application point of view, and do not investigate the benefits of multipath streaming. The advantage of user-level channel diversity is studied in [26] in terms of performance, fairness, robustness and cost. On a different client-server scenario, we achieve the optimization of the received media quality, when the available network resources for the application represent the share of the total resources split among concurrent applications.

More generally, routing with multiple metrics is the target of many works in QoS routing. But QoS routing with multiple constraints is, in general, an NP hard problem. An initial proof, for the case of at least two additive metrics is given in [27]. The authors propose heuristic algorithms for both source routing, and hop-by-hop routing, which find one path satisfying the QoS requirements of multimedia applications. Recent works in multiconstrained routing optimize a linear [28], respectively a nonlinear [29] relation between constraints, using low complexity algorithms. A similar function built on multiple path metrics is used in [30] to find multiple network paths for streaming.

In contrary to common QoS routing problems, we propose a media-specific distortion metric, which comprises multiple network link parameters together with media aware parameters. The metric describes the quality of the received video, as a function of the specific network scenario and streaming process. The optimization of the end-to-end distortion translates into choosing the best set of paths, and the respective optimal 
rate allocation. Classical optimization methods however fail to obtain a simple solution due to the nonconvexity of the optimization function. An in-depth analysis of the behavior of this metric in our specific setting however, allows to derive a simple algorithm that achieves the optimal solution in linear time, as it has been shown in this paper.

Flow assignment problems have been addressed in [31] and [32]. The authors of the first paper are concerned with optimally splitting the data on multiple disjoint paths in order to avoid packet re-sequencing at the client. The second paper presents an algorithm that minimizes the end-to-end delay of data transmission while complying with an aggregated bandwidth constraint. The algorithm is optimal only in the case of unit capacity links and disjoint paths. Our flow problem formulation is general and deals with both joint and disjoint paths. We show that, from the media application point of view, an optimal flow allocation is achievable in any network scenario, by joint optimization of the number of paths used, and the aggregated rate of the flows.

Finally, the multipath problem is specifically addressed in the case of media streaming in [33]. The authors present a FEC scheme combined with server diversity and a packet scheduling mechanism, which intends to minimize the cumulative distortion of individual erroneous video packets. Our work focuses on a nonmulticast communication scenario, with an intermediate network comprising multiple available transmission paths. Multistream coding, combined with multipath transmission, has been presented in [34] as a solution to fight against network errors in an ad-hoc network environment. In the same time, the authors of [35] analyze a multiple path streaming scenario for the transmission of a video sequences encoded in multiple descriptions. They minimize an additive distortion metric, computed as the sum of the individual distortions of each of the independent descriptions. For complexity reasons, their analysis is reduced to a scenario comprising two encoded descriptions and two transmission paths. In our work we rather address the questions of how many transmission paths to use, and how to chose them, in order to maximize the efficiency of the streaming application. Our streaming framework is more general, and applicable to any streaming scenario that obeys an additive rule for the aggregated transmitted rate and loss process. The proposed algorithm finds the optimal transmission strategy and encoding rate, based only on the available network resources, and video sequence dependent parameters.

\section{CONCLUSIONS}

In this paper, we propose to use a flow model to analyze the opportunity of multipath media streaming over the Internet. Based on an equivalent transformation between the available network graph and a tree of flows, we jointly determine the network paths, and the optimal rate allocation for generic streaming scenarios. A media specific performance metric is used, which takes into account the end-to-end network path parameters along with media aware parameters.

An in-depth analysis of the end-to-end distortion behavior, in the given network scenario, drives the design of a linear time algorithm for optimal rate allocation. The form of the optimal rate allocation solution follows a simple greedy rule that always uses the paths with the lowest loss probability first. In particular, we show that extra network paths are either used at their maximum available bandwidth, if their value is large enough, or simply ignored. The overall rate allocation solution offers a careful trade-off between extra transmission rate and increase in the end-to-end error process. Even for large network scenarios, only a small number of paths should optimally be used for transmission, and they should be chosen among the lowest loss probability channels.

The optimal rate allocation algorithm has been tested in various random network scenarios, and it significantly outperforms simpler schemes based on heuristic rate allocation strategies. In many cases, our algorithm even provides an end-to-end distortion improvement of more than 20\%. Due to its low complexity, and important benefits in most streaming scenarios, the optimal rate allocation algorithm provides a very interesting solution to efficient media streaming over resource-constrained networks.

\section{REFERENCES}

[1] L. Golubchik, J. Lui, T. Tung, A. Chow, and W. Lee, "Multi-path continuous media streaming: What are the benefits," ACM J. Perform. Eval., vol. 49, no. 1-4, pp. 429-449, Sep. 2002.

[2] Y. Li, S. Mao, and S. S. Panwar, "The case for multipath multimedia transport over wireless ad hoc networks," in Proc. IEEE/ACM BroadNets, Oct. 2004, pp. 486-495.

[3] T. Nguyen and A. Zakhor, "Distributed video streaming with forward error correction," in Proc. Packet Video Workshop, Pittsburgh, PA, 2002.

[4] J. G. Apostolopoulos and M. D. Trott, "Path diversity for enhanced media streaming," IEEE Commun. Mag., vol. 42, no. 8, pp. 80-87, Aug. 2004.

[5] ITU, Recommend. H.264 Mar. 2005

[6] H. M. Radha, M. van der Schaar, and Y. Chen, "The mpeg-4 finegrained scalable video coding method for multimedia streaming over ip," IEEE Trans. Multimedia, vol. 3, no. 1, pp. 53-68, Mar. 2001.

[7] S. Savage, A. Collins, and E. Hoffman, "The end-to-end effects of internet path selection," in Proc. ACM SIGCOMM, 1999, pp. 289-299.

[8] J. Apostolopoulos, T. Wong, W. Tan, and S. Wee, "On multiple description streaming with content delivery networks," in Proc. IEEE INFOCOM, Jun. 23-27, 2002, vol. 3, pp. 1736-1745.

[9] P. Frossard and O. Verscheure, "Joint source/fec rate selection for quality-optimal MPEG-2 video delivery," IEEE Trans. Image Processing, vol. 10, no. 12, pp. 1815-1825, 2001.

[10] D. Jurca, S. Petrovic, and P. Frossard, "Media aware routing in large scale networks with overlay," in Proc. IEEE ICME, July 2005.

[11] Y. J. Liang, J. G. Apostolopoulos, and B. Girod, "Analysis of packet loss for compressed video: Does burst-length matter," in Proc. IEEE ICASSP, 2003.

[12] K. Stuhlmuller, N. Farber, M. Link, and B. Girod, "Analysis of video transmission over lossy channels," IEEE J. Select. Areas Commun., vol. 18, no. 6, pp. 1012-1032, Jun. 2000.

[13] M. Dai and D. Loguinov, "Analysis of rate-distortion functions and congestion control in scalable internet video streaming," in NOSSDAV '03: Proc. 13th Int. Workshop on Network and Operating Systems Support for Digital Audio and Video, New York, 2003, pp. 60-69.

[14] Y. J. Liang, J. G. Apostolopoulos, and B. Girod, "Analysis of packet loss for compressed video: Does burst-length matter," in Proc. IEEE ICASSP, Apr. 2003, vol. 5, pp. 687-884.

[15] Z. Michalewicz and D. B. Fogel, How to Solve It: Modern Heuristics, 2nd ed. New York: Springer-Verlag, 2000.

[16] V. Ribeiro, R. Riedi, R. Baraniuk, J. Navratil, and L. Cottrell, "PathChirp: Efficient available bandwidth estimation for network paths," in Proc. Passive and Active Measurement Workshop, Apr. 2003.

[17] P. von Rickenbach, S. Schmid, R. Wattenhofer, and A. Zollinger, "A robust interference model for wireless ad-hoc networks," in Proc. IEEE WMAN'05, 2005

[18] R. Chandra, P. Bahl, and P. Bahl, "Multinet: Connecting to multiple ieee 802.11 networks using a single wireless card," in Proc. IEEE INFOCOM, 2005, vol. 2, pp. 882-893. 
[19] G. Epshtein, "A better way to implement video over WLAN," Metalink-White Paper 2005.

[20] Swisscom Mobile Unlimited UMTS/GPRS/WLAN [Online]. Available: http://www.swisscom-mobile.ch/scm/gek_mobile-unlimited-en.aspx

[21] D. Jurca and P. Frossard, "Distributed media rate allocation in overlay networks," in Proc. IEEE ICME, Jul. 2006.

[22] D. Jurca and P. Frossard, "Video packet selection and scheduling for multipath streaming," IEEE Trans. Multimedia, vol. 9, no. 3, pp. 629-641, Apr. 2007.

[23] S. Vutukury and J. J. Garcia-Luna-Aceves, "MDVA: A distance-vector multipath routing protocol," in Proc. IEEE INFOCOM, 2001, vol. 1, pp. 557-564.

[24] W. Wei and A. Zakhor, "Multipath unicast and multicast video communication over wireless ad hoc networks," in Proc. IEEE/ACM BroadNets, Oct. 2004, pp. 496-505.

[25] S. Tao and R. Guerin, "Application-specific path switching: A case study for streaming video," in Proc. ACM Multimedia, Oct. 2004, pp. 136-143.

[26] E. Vergetis, R. Guerin, and S. Sarkar, "Realizing the benefits of userlevel channel diversity," ACM SIGCOMM Comput. Commun. Rev., vol. 35 , no. 5, pp. 15-28, Oct. 2005.

[27] Z. Wang and J. Crowcroft, "Quality-of-service routing for supporting multimedia applications," IEEE J. Select. Areas Commun., vol. 14, no. 7, pp. 1228-1234, Sep. 1996.

[28] Y. Cui, K. Xu, and J. Wu, "Precomputation for multi-constrained qos routing in high-speed networks," in Proc. IEEE INFOCOM, 2003, vol. 2, pp. 1414-1424.

[29] T. Korkmaz and M. M. Krunz, "Routing multimedia traffic with qos guarantees," IEEE Trans. Multimedia, vol. 5, no. 3, pp. 429-443, Sep. 2003.

[30] Z. Ma, H.-R. Shao, and C. Shen, "A new multi-path selection scheme for video streaming on overlay networks," in Proc. IEEE ICC, 2004.

[31] K. C. Leung and V. O. K. Li, "Flow assignement and packet scheduling for multipath routing," J. Commun. Networks, vol. 5, no. 3, pp. 230-239, Sep. 2003.

[32] J. Chen, S.-H. G. Chan, and V. O. K. Li, "Multipath routing for video delivery over bandwidth-limited networks," IEEE J. Select. Areas Commun., vol. 22, no. 10, pp. 1920-1932, Dec. 2004.

[33] T. Nguyen and A. Zakhor, "Path diversity with forward error correction (pdf) system for packet switched networks," in Proc. IEEE INFOCOM, 2003, vol. 3, pp. 663-672.

[34] S. Mao, S. Lin, S. S. Panwar, Y. Wang, and E. Celebi, "Video transport over ad hoc networks: Multistream coding with multipath transport," IEEE J. Select. Areas Commun., vol. 21, no. 10, pp. 1721-1737, Dec. 2003.

[35] A. C. Begen, Y. Altunbasak, O. Ergun, and M. H. Ammar, "Multipath selection for multiple description video streaming over overlay networks," Signal Proces.: Image Commun., vol. 20, pp. 39-60, 2005.

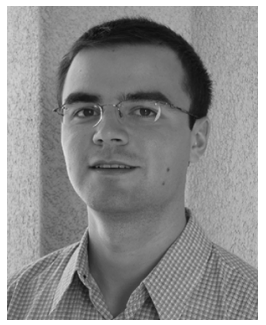

Dan Jurca (S'03) received the B.Sc. degree from the "Politehnica" University of Timsoara, Romania, in 2002. In 2003, he graduated from the Graduate School in Computer and Communication Sciences, Ecole Politechnique Federale de Lausanne (EPFL), Switzerland, where he is currently pursuing the Ph.D. degree.

His research interests focus on adaptive rich media streaming (forward error correction, multipath media transmissions, and streaming in wireless environments).

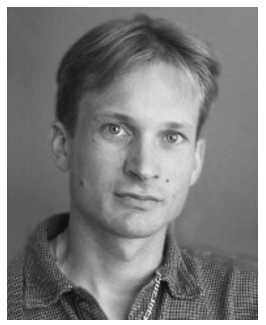

Pascal Frossard (S'96-M'01-SM'04) received the M.S. and Ph.D. degrees, both in electrical engineering, from the Swiss Federal Institute of Technology (EPFL), Lausanne, Switzerland, in 1997 and 2000, respectively. From 1998 to 2000, he was with the Signal Processing Laboratory, EPFL, as a Research and Teaching Assistant under a grant from Hewlett-Packard.

Between 2001 and 2003, he was a Member of Research Staff at the IBM T. J. Watson Research Center, Yorktown Heights, NY, where he worked on media compression and streaming technologies. Since 2003, he has been an Assistant Professor at EPFL, supported by the Swiss National Science Foundation. His research interests include image representation and coding, nonlinear representations, visual information analysis, joint source and channel coding, multimedia communications, and multimedia content distribution.

Dr. Frossard has been the General Chair of IEEE ICME 2002, Lausanne, and member of the organizing or technical program committees of numerous conferences. He has served as Guest Editor of special issues on streaming media (IEEE TRANSACTIONS ON MULTIMEDIA), on media and communication applications on general purpose processors: hardware and software issues (Journal of VLSI SPSS), and on image and video coding beyond standards (Journal of Signal Processing). He is an Associate Editor of the IEEE TRANSACTIONS ON MULTIMEDIA (2004-present) and of the IEEE TRANSACTIONS ON CIRCUITS AND SYSTEMS FOR VIDEO TECHNOLOGY (2006-present), and he served as a member of the Editorial Board of the EURASIP Journal of Signal Processing (2003-2005). Since 2004, he has served as Vice-Chair of the IEEE Multimedia Communications Technical Committee, as a member of the IEEE Multimedia Signal Processing Technical Committee, and of the IEEE Multimedia Systems and Applications Technical Committee. He received the Swiss NSF Professorship Award in 2003, and the IBM Faculty Award in 2005. 